\title{
ATM deficiency induces oxidative stress and endoplasmic reticulum stress in astrocytes
}

\author{
Na Liu ${ }^{1}$, George Stoica ${ }^{2}$, Mingshan Yan ${ }^{1}$, Virginia L Scofield ${ }^{1}$, Wenan Qiang ${ }^{3}$, \\ William S Lynn ${ }^{1}$ and Paul KY Wong ${ }^{1}$ \\ ${ }^{1}$ Department of Carcinogenesis, The University of Texas MD Anderson Cancer Center, Science Park-Research \\ Division, Smithville, TX, USA; ${ }^{2}$ Department of Pathology, Texas A\&M University, College Station, TX, USA \\ and ${ }^{3}$ Department of Cell and Molecular Biology, Northwestern University Feinberg School of Medicine, \\ Chicago, IL, USA
}

\begin{abstract}
ATM kinase, the product of the ataxia telangiectasia mutated $($ Atm $)$ gene, is activated by genomic damage. ATM plays a crucial role in cell growth and development. Here we report that primary astrocytes isolated from ATMdeficient mice grow slowly, become senescent, and die in culture. However, before reaching senescence, these primary $\mathrm{Atm}^{--}$astrocytes, like $\mathrm{Atm}^{--}$lymphocytes, show increased spontaneous DNA synthesis. These astrocytes also show markers of oxidative stress and endoplasmic reticulum (ER) stress, including increased levels of heat shock proteins (HSP70 and GRP78), malondialdehyde adducts, Cu/Zn superoxide dismutase, procaspase 12 cleavage, and redox-sensitive phosphorylation of extracellular signal-regulated protein kinase 1 and 2 (ERK1/2). In addition, HSP70 and ERK1/2 phosphorylation are upregulated in the cerebella of ATMdeficient mice. This increase in ERK1/2 phosphorylation is seen primarily in cerebellar astrocytes, or Bergmann glia, near degenerating Purkinje cells. ERK1/2 activation and astrogliosis are also found in other parts of the brain, for example, the cortex. We conclude that ATM deficiency induces intrinsic growth defects, oxidative stress, ER stress, and ERKs activation in astrocytes.
\end{abstract}

Laboratory Investigation (2005) 85, 1471-1480. doi:10.1038/labinvest.3700354; published online 26 September 2005

Keywords: ataxia telangiectasia mutated; oxidative stress; extracellular signal-regulated protein kinases; endoplasmic reticulum stress; astrocyte; cerebellar Bergmann glia

Ataxia telangiectasia (A-T) is a human genetic disease whose hallmarks are oxidative damage, genomic instability, neurodegeneration, immunodeficiency, premature aging, sterility, and a predisposition to cancer. ATM, the product of the A-T mutated gene $($ Atm $)$ is a large, multifunctional kinase that regulates responses required for cellular survival in response to DNA double-strand breaks caused by ionizing radiation, DNA damage agents, and DNA recombination. ATM undergoes autophosphorylation and subsequently initiates phosphorylation of multiple substrates, many of which are cell cycle regulators, such as p53. Activated ATM leads to cell cycle arrest, and also functions in concert with factors that repair DNA damage..$^{1-4}$

Correspondence: Professor PKY Wong, PhD, Department of Carcinogenesis, The University of Texas MD Anderson Cancer Center, Science Park-Research Division, Smithville, TX 78957, USA.

E-mail: pkwong@mdanderson.org

Received 7 May 2005; revised 9 August 2005; accepted 12 August 2005; published online 26 September 2005
In addition to its functions in repairing DNA damage induced by deleterious insults, ATM may be involved in sensing and modulating intracellular redox status. ATM helps hematopoietic stem cells maintain the capacity for self-renewal under oxidative stress. ${ }^{5}$ ATM deficiency causes oxidative damage to proteins and lipids in the brain, testes, and thymus. ${ }^{6}$ Several other studies have confirmed that the absence of ATM results in oxidative stress in the brain. ${ }^{7-9}$

A- $\mathrm{T}$ produces severe neurodegeneration in humans, with ataxia and Purkinje cell damage. Several studies have shown that $\mathrm{Atm}^{-1-}$ mice exhibit signs of A-T-like neurodegeneration, including oxidative stress in Purkinje cells and substantia nigral dopaminergic neurons, ${ }^{10}$ but no ataxia-like movement disorder. It has been shown that ATM deficiency in the $\mathrm{Atm}^{-1-}$ mouse brain results postnatally in deficient dendritogenesis in Purkinje neurons ${ }^{9}$ as well as reduced cell size and diminished cell density in substantia nigral dopaminergic neurons. ${ }^{8,10} \mathrm{Atm}^{-1-}$ mice also show 'predegenerative' CNS lesions with age-dependent neuronal cell loss and gliosis, ${ }^{11}$ dystrophic Purkinje cells, ${ }^{12}$ and 
indicators of Purkinje cell cycle re-entry postnatally. ${ }^{13}$ It has also been reported that in vitro survival of cerebellar Purkinje cells from Atm $^{-1-}$ mice is significantly reduced compared with their wild-type littermates. ${ }^{9}$ Taken together, these reports show that Purkinje cells and substantia nigral dopaminergic neurons are damaged both in A-T humans and in ATM-deficient mice, although neurobehavioral consequences of these changes are less evident in $\mathrm{Atm}^{-1-}$ mice. Antioxidant treatments have been shown to correct some neurobehavioral defects in $\mathrm{Atm}^{-1-}$ mice $^{8}$ and to enhance $\mathrm{Atm}^{-1-}$ Purkinje cell survival and dendritogenesis in culture. ${ }^{9}$

Neurons are particularly vulnerable to oxidative stress, and neurons depend on astrocytes for antioxidant support. ${ }^{14-17}$ Loss of astrocytic antioxidant support may contribute to neuronal death. ${ }^{18-23}$ The findings of one study suggest that $\mathrm{Atm}^{-1-}$ astrocytes are unable to protect Purkinje cells against oxidative stress. ${ }^{9}$ However, it is not clear how Atm $^{-1-}$ astrocytes fail to protect these neurons.

Our previous study showed that Atm $^{-1-}$ thymocytes exhibit increased spontaneous DNA synthesis, which is associated with development of thymic lymphoma, and that by suppressing this redoxsensitive DNA synthesis thymic lymphoma can be prevented. $^{24-26}$ This suggests that ATM may control cell cycling by modulating redox status. ${ }^{27}$ We show here that primary $\mathrm{Atm}^{-1-}$ astrocytes in culture, which exibit growth abnormality, also show increased spontaneous DNA synthesis and markers of oxidative stress and endoplasmic reticulum (ER) stress. In addition, activation of redox-sensitive extracellular signal-regulated protein kinase 1 and 2 (ERK1/2) is increased in primary $\mathrm{Atm}^{-/}$astrocytes and in Bergmann glia (astrocytes next to Purkinje neurons) in the cerebella of $\mathrm{Atm}^{-1-}$ mice. We also detect ERK1/2 activation in cortical neurons and astrogliosis in cortices of $\mathrm{Atm}^{-1-}$ mice. Thus, this study indicates ERK1/2 activation in both $\mathrm{Atm}^{-1}$ astrocytes and neurons, and suggests that oxidatively stressed $\mathrm{Atm}^{-1-}$ astrocytes may partially contribute to the failure of antioxidant support to the neurons, eventually leading to neuronal oxidative stress.

\section{Materials and methods}

\section{Materials}

DMEM/F12 medium, fetal bovine serum (FBS), penicillin, streptomycin, Fungizone, and trypsin were purchased from Invitrogen Life Technologies (Carlsbad, CA, USA). All other reagents, unless otherwise specified, were purchased from Sigma Chemical Co. (St Louis, MO, USA).

\section{Animals}

The $\mathrm{Atm}^{-1-}$ mice were developed by Barlow et al. ${ }^{6}$ Heterozygous $\mathrm{Atm}^{+/-}$mice were purchased from
Jackson Laboratory (Bar Harbor, ME, USA) and kept in the Animal Center at Science Park-Research Division of The University of Texas MD Anderson Cancer Center under standard conditions approved by the IACUC. These $\mathrm{Atm}^{+/-}$mice were mated to produce newborns (total 40 pups) for primary cultures, and mature mice ( 2 or 3 months old, 10 $\mathrm{Atm}^{+/+}$and $8 \mathrm{Atm}^{-/-}$) were used for immunoblotting and immunohistochemistry.

\section{Primary Culture of Astrocytes}

Primary astrocytes were isolated from 1- to 2-dayold newborn mouse pups by a method described previously. ${ }^{28}$ Pups from heterozygous $\left(\mathrm{Atm}^{+/}\right)$ parents were killed by carbon dioxide. The cerebellum of each newborn mouse was removed and minced separately in ice-cold DMEM/F12 medium and a small piece of skin from the ear pouch of each newborn was collected and kept for future DNA extraction and genotyping. A single-cell suspension was obtained by passing the minced cerebellar tissue through a $70-\mu \mathrm{m}$ nylon mesh cell strainer and plated onto poly-L-lysine-coated 35-mm flasks and grown in DMEM/F12 medium, supplemented with $10 \%$ FBS, $100 \mathrm{U} / \mathrm{ml}$ penicillin, $100 \mu \mathrm{g} / \mathrm{ml}$ streptomycin, and $2.5 \mu \mathrm{g} / \mathrm{ml}$ Fungizone for 7-15 days until reaching confluence. ${ }^{29}$ The cells developed into (80-85\%) enriched astroglial cell cultures after passage in vitro at $37^{\circ} \mathrm{C}$ in $5 \%$ $\mathrm{CO}_{2}$. Anti-glial fibrillary acidic protein (anti-GFAP) was used to identify astrocytes. Pup genotypes $\left(\mathrm{Atm}^{+/+}, \mathrm{Atm}^{+/-}\right.$, or $\left.\mathrm{Atm}^{-1-}\right)$ were then identified by using standard genotyping protocol on ear pouch skin samples and used to label the respective primary culture cells. Astrocytes were allowed to grow to confluence and were used for flow cytometry, thymidine uptake, and immunoblotting analyses.

\section{Flow Cytometry}

Cultured cells at confluence were gently trypsinized and removed from the culture dishes. The suspended cells were fixed in $70 \%$ ethanol and stained with propidium iodide containing RNase (DNasefree; Roche, Indianapolis, IN, USA). DNA content was then analyzed on a Coulter Epics Elite flow cytometer (Beckman-Coulter, Miami, FL, USA). Cell cycle analysis was performed with the Multicycle system (Phoenix Flow Systems, San Diego, CA, USA).

\section{$\left[{ }^{3} \mathrm{H}\right]$ thymidine Uptake}

Astrocytes were seeded in 24-well plates at a concentration of $1 \times 10^{5}$ cells/well and fed with 40 , 10 or $0.5 \%$ FBS medium for 2 days. $\left[{ }^{3} \mathrm{H}\right]$ thymidine $(2 \mu \mathrm{Ci})$ was added $16 \mathrm{~h}$ before the cells were 
harvested for radioactivity counting. Results were expressed as mean counts per minute per $10^{4}$ cells \pm standard deviation in triplicate cultures.

\section{Immunoblotting}

Astrocytes collected from cultures and cerebella isolated from $\mathrm{CO}_{2}$-sacrificed mice were harvested, and total proteins were extracted by using RIPA buffer ( $1 \%$ NP40, $0.5 \%$ sodium deoxycholate, $0.1 \%$ sodium dodecyl sulfate, $0.25 \mathrm{mM}$ phenylmethylsulfonyl fluoride, and $1 \mathrm{mM}$ sodium fluoride in phosphate-buffered saline), supplemented with a protease inhibitor cocktail (Sigma), according to a modified protocol described previously. ${ }^{29}$ Protein concentrations were measured by using Bio-Rad $D C$ Protein Assay Reagent (Bio-Rad Laboratories, Hercules, CA, USA) following the manufacturer's suggestions. In all $75 \mu \mathrm{g}$ of protein from cell lysates (or $50 \mu \mathrm{g}$ for tissue lysates) was separated by $12 \%$ sodium dodecyl sulfate-polyacrylamide gel electrophoresis. After electrophoresis, the proteins were transferred to a polyvinylidene fluoride membrane (Millipore Corp., Billerica, MA, USA). The membrane was blocked for $1 \mathrm{~h}$ in Tris-buffered saline

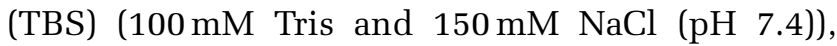
with $5 \%$ nonfat milk at room temperature and then immunoblotted with antibodies against procaspase12 (BioVision Inc., Mountain View, CA, USA); ERK1/2 and phosphorylated ERK1/2 (Cell Signaling Technology Inc., Beverly, MA, USA); GRP78 and heat shock protein (HSP) 70 (Santa Cruz Biotechnology, Santa Cruz, CA, USA); and MDA (Alpha Diagnostic, San Antonio, TX, USA). Afterward, the membranes were incubated with horseradish peroxidase-conjugated secondary antibodies, and immune complexes were detected by chemiluminescence (NEN Life Science Products, Inc., Boston, MA, USA).

\section{Immunohistochemistry}

Paraffin-embedded sections ( $6 \mu \mathrm{m}$ thick) of formalinfixed 2-month-old mouse brains (four of $\mathrm{Atm}^{+/+}$, three of $\mathrm{Atm}^{-1-}$ ) and 3-month-old mouse brains (three of $\mathrm{Atm}^{+/+}$, three of $\mathrm{Atm}^{-1-}$ ) were deparaffinized and washed with TBS for $20 \mathrm{~min}$ at room temperature. Potential nonspecific binding sites were blocked with $5 \%$ normal goat serum in TBS, after which the tissue sections were incubated with phospho-p44/42 MAPK (ERK1/2) rabbit monoclonal antibody (Cell Signaling Technology Inc., Beverly, MA, USA) at a dilution of 1:100 for $4 \mathrm{~h}$. After three 5 -min washes in TBS, the sections were then incubated with biotin-conjugated secondary antirabbit IgG (Vector Laboratories, Burlingame, CA, USA) for $1 \mathrm{~h}$ at room temperature. A Vecta-Elite streptavidin-peroxidase kit with an alkaline phosphatase substrate was used for color development. The sections were counterstained with diluted hematoxylin. Sections that were not incubated with the primary antibody served as negative controls. Labeling of Bergman glial cells was performed according to a previously published method. ${ }^{30} \mathrm{~S}$ 100B monoclonal antibody (diluted 1:4000) was purchased from Sigma-Aldrich, St Louis, MO, USA) and immunohistochemistry was carried out on consecutive sections of the cerebellum. Control sections were incubated with normal sera instead of the primary antibody, followed by all subsequent incubations. No nonspecific staining was observed. Sections were examined and photographed using an Olympus BH-2 microscope.

\section{Statistical Analysis}

Data are presented as mean \pm standard deviation. The statistical significance of the results was determined by Student's $t$-test. A probability of less than $5 \%$ was considered significant.

\section{Results}

\section{Growth Abnormality and Increased Spontaneous DNA Synthesis in Atm $^{-1-}$ Astrocytes}

The astrocytes isolated from $\mathrm{Atm}^{-1-}$ mice grow more slowly in $10 \%$ FBS than do those from $\mathrm{Atm}^{+/+}$and $\mathrm{Atm}^{+/-}$mice, as previously reported by Gosink et $\mathrm{al}^{31}{ }^{31} \mathrm{In}$ our study, the average time for $\mathrm{Atm}^{-/-}$ astrocytes to reach confluence was prolonged in medium containing 10\% FBS (Figure 1a, $P<0.01$ ). When cultured in medium containing $0.5 \%$ FBS, $\mathrm{Atm}^{-1-}$ astrocytes failed to reach confluence and rapidly died. In contrast, $\mathrm{Atm}^{+/+}$and $\mathrm{Atm}^{+/-}$ astrocytes continued to proliferate in medium containing $0.5 \%$ FBS and reached confluence (data not shown). In medium containing 40\% FBS, $\mathrm{Atm}^{-1-}$ cells proliferated slightly faster than those cultured in $10 \%$ FBS and reached confluence, but at slower rates than $\mathrm{Atm}^{+/+}$and $\mathrm{Atm}^{+/-}$astrocytes (data not shown). In the later studies shown here, in order to obtain samples from both $\mathrm{Atm}^{+/+}$and $\mathrm{Atm}^{-1-}$, we only used medium containing 10\% FBS for both genotypes. And we did not use $\mathrm{Atm}^{+/-}$ astrocytes in parallel with $\mathrm{Atm}^{+/+}$and $\mathrm{Atm}^{-1-}$ astrocyte, since there is no significant difference of cell growth between $\mathrm{Atm}^{+/+}$and $\mathrm{Atm}^{+/-}$astrocytes. $\mathrm{Atm}^{-1-}$ astrocytes grown in $10 \%$ FBS could be passed only 3-4 times before they became flat and enlarged with increased intracellular vacuolation, ceased proliferating, and died (Figure 1b). In contrast, $\mathrm{Atm}^{+/+}$and $\mathrm{Atm}^{+/-}$astrocytes could be passed more than 12 times.

We have shown previously that ATM-deficient murine thymocytes and human lymphocytes survive in culture only with high concentrations of serum, and that the rates of spontaneous DNA synthesis are greatly increased. ${ }^{26,32}$ To determine whether this also occurs in $\mathrm{Atm}^{-1-}$ astrocytes, we 

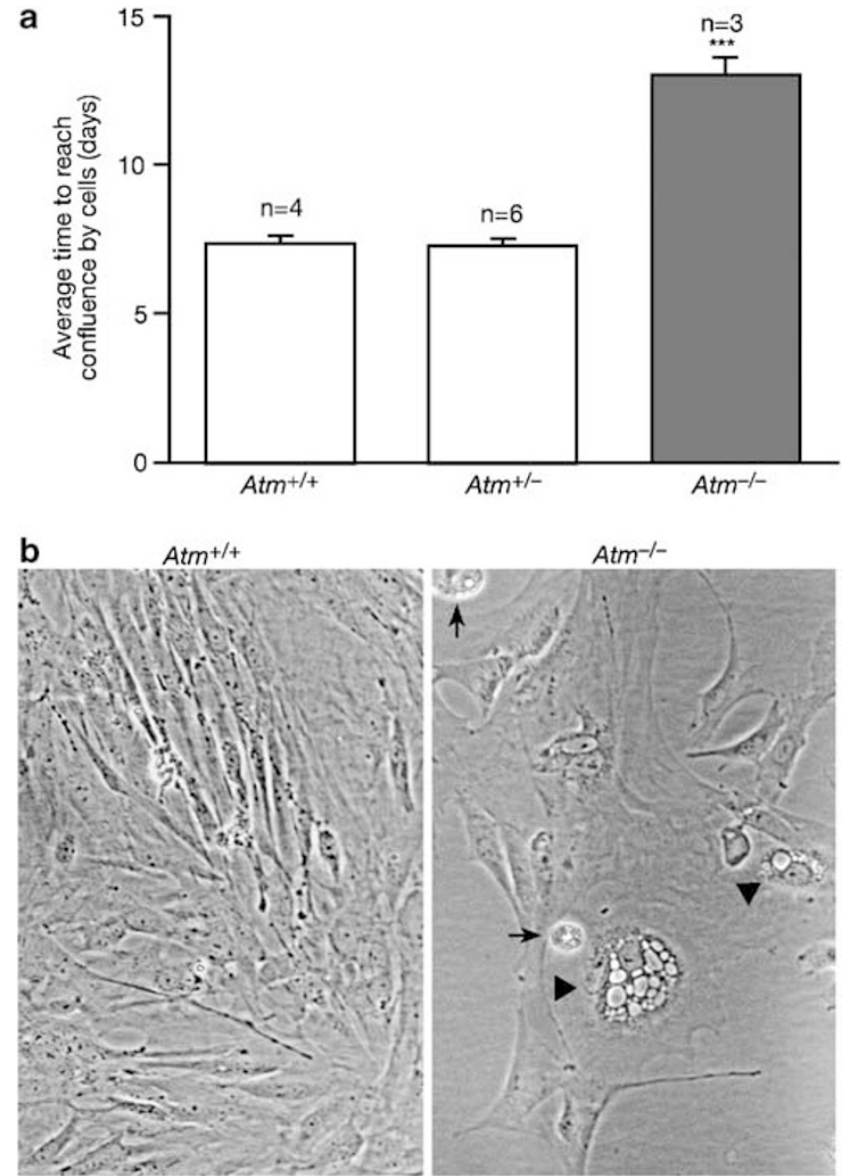

Figure 1 The growth of Atm astrocytes in culture. Newborn mice were used for harvesting of primary cultures of astrocytes and genotyping. Primary culture from each pup was plated into one poly-L-lycine-coated 35-mm flask, and supplemented with medium containing $10 \%$ FBS. (a) The number of days from plating until the primary culture reached complete confluence was recorded as the first passage time. The graph compares the average time of first passage of cultures from four $\mathrm{Atm}^{+/+}$pups, six $\mathrm{Atm}^{+/-}$pups, and three $\mathrm{Atm}^{-1-}$ pups. ${ }^{* * *}$ signifies $P<0.001$, average time of $\mathrm{Atm}^{+/+}$vs that of $\mathrm{Atm}^{-/-}$. (b) The morphologic difference between primary cultures of $\mathrm{Atm}^{+/+}$and $\mathrm{Atm}^{-/-}$ astrocytes. After four passages under identical culture conditions, $\mathrm{Atm}^{+/+}$astrocytes proliferated and reached confluence. Atm ${ }^{-\prime-}$ astrocytes, however, could not reach confluence, and showed increased body size and intracellular vacuolization (arrowheads) and an increased number of dead cells (arrows).

compared DNA synthesis and cell cycle phase in cultures of astrocytes from $\mathrm{Atm}^{+/+}$and $\mathrm{Atm}^{-1-}$ mice after the first passage. The rate of $\left[{ }^{3} \mathrm{H}\right]$ thymidine uptake by $\mathrm{Atm}^{-/-}$astrocytes was markedly higher than that of $\mathrm{Atm}^{+/+}$astrocytes at all serum concentrations (Figure 2a). Cell cycle analysis showed that in medium containing $10 \%$ FBS, $\mathrm{Atm}^{-1-}$ and $\mathrm{Atm}^{+/+}$astrocytes were cycling at similar rates and were mostly in the G1 phase at confluence (Figure $2 \mathrm{~b}$ ). The rate of $\left[{ }^{3} \mathrm{H}\right]$ thymidine uptake was also greater at lower FBS concentrations in both $\mathrm{Atm}^{-1-}$ and $\mathrm{Atm}^{+/+}$astrocytes, although $\left[{ }^{3} \mathrm{H}\right]$ thymidine uptake was much higher in the former.

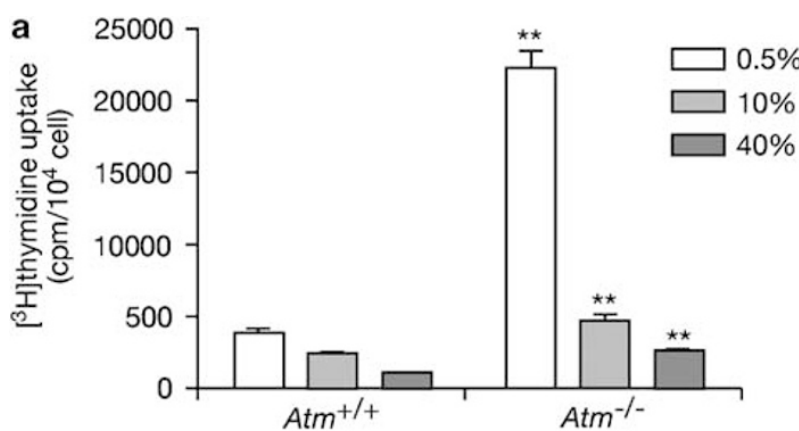

b

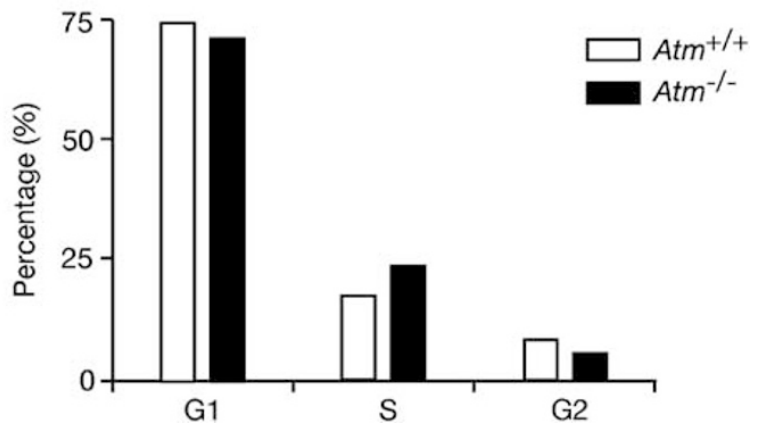

Figure 2 DNA synthesis and cell cycle analysis in primary cultures of $\mathrm{Atm}^{+/+}$and $\mathrm{Atm}^{-1-}$ astrocytes. (a) Primary cultures in 35-mm flasks were passed once for $\mathrm{Atm}^{-1-}$ astrocytes and twice for $\mathrm{Atm}^{+/+}$astrocytes before being seeded into 24-well plates at a concentration of $1 \times 10^{5}$ cells/well and fed overnight with medium containing $40 \%$ FBS. Then the medium was exchanged for new medium containing 40,10 or $0.5 \%$ FBS for 2 days. $\left[{ }^{3} \mathrm{H}\right]$ thymidine $(2 \mu \mathrm{Ci} /$ well) was added $16 \mathrm{~h}$ before harvesting the cells for radioactivity counting and cell counting. The graph shows the average $\left[{ }^{3} \mathrm{H}\right]$ thymidine uptake from triplicate experiments, normalized by total cell number. **identifies $P<0.01$, average counting of $\mathrm{Atm}^{+/+}$vs that of $\mathrm{Atm}^{-1-}$. (b) Primary cultures in 60-mm flasks were passed once for $\mathrm{Atm}^{-1-}$ astrocytes and twice for $\mathrm{Atm}^{+/+}$astrocytes before being maintained in medium containing 10\% FBS until confluent and then harvested for propidium iodide staining and cell cycle analysis. Both $\mathrm{Atm}^{+/+}$and $\mathrm{Atm}^{-1-}$ astrocytes were arrested in the G1 phase. The same blot was stripped and immunoblotted with $\beta$-actin antibody.

\section{Atm-Deficient Astrocytes Show Signs of Oxidative Stress and ER Stress in Culture}

Signs of oxidative stress are present in $\mathrm{Atm}^{-/-}$ mouse brains and neurons. ${ }^{6-9}$ As astrocytes normally provide trophic and antioxidant support for neurons, ${ }^{17,33-36}$ it is possible that $\mathrm{Atm}^{-/-}$astrocytes are oxidatively stressed and unable to provide adequate antioxidant support to $\mathrm{Atm}^{-1-}$ neurons, which may lead to oxidative stress in nearby neurons with resultant neuronal degeneration.

In our study, some markers of oxidative stress, including HSP70, $\mathrm{Cu} / \mathrm{ZnSOD}$, and MnSOD were increased in $\mathrm{Atm}^{-1-}$ astrocytes (Figure 3a). Malondialdehyde (MDA) is a byproduct of lipid peroxidation and readily combines with proteins, lipoproteins, and DNA. The detection of MDAmodified proteins shows the end point of lipid peroxidation and is used as a marker of oxidative damage. ${ }^{37}$ In our study, the amounts of (MDA)protein adducts at $\sim 68-, 50-, 37-$ and $30-\mathrm{kDa}$ were 
a

Atm
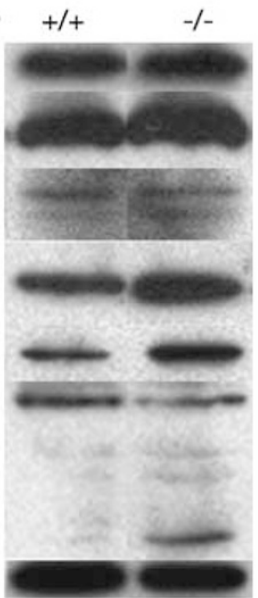

MnSOD

$\mathrm{Cu} / \mathrm{ZnSOD}$

Catalase

HSP70

GRP78

pro $\longrightarrow$

cleaved

$\beta$-actin

c
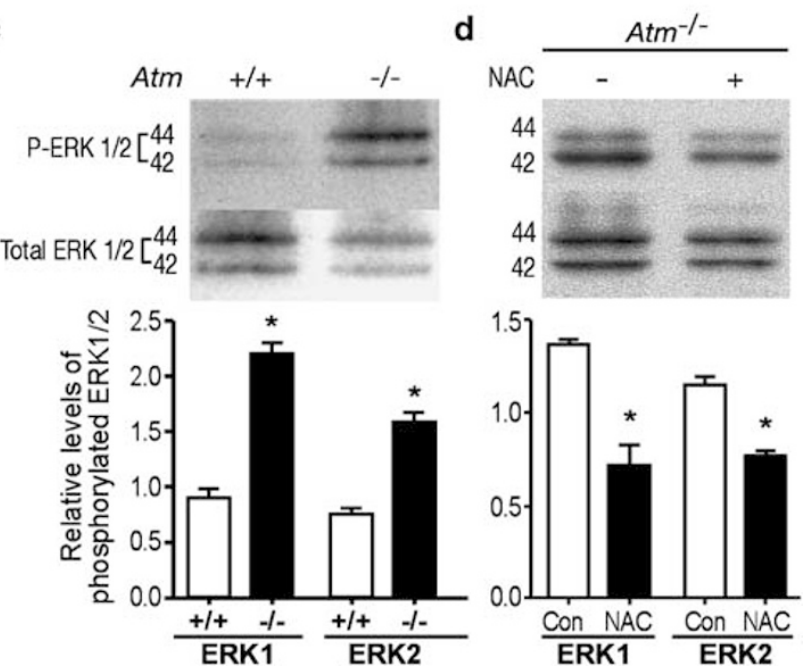

Figure 3 Oxidative stress, ER stress, and ERK1/2 activation in $\mathrm{Atm}^{-1-}$ astrocytes. After the initial passage, three combined cultures of $\mathrm{Atm}^{+/+}$astrocytes and three of $\mathrm{Atm}^{-/-}$astrocytes were cultured until they reached confluence. The combined cultures were then harvested for Western blotting. Bands from Western blotting were scanned by Kodak Image Station for further analysis. The graphs represent the results of two or more separate experiments. (a) The bands of $\mathrm{MnSOD}, \mathrm{Cu} / \mathrm{ZnSOD}$, catalase, HSP70, GRP78, and caspase 12 from astrocytes of $\mathrm{Atm}^{+/+}$and $\mathrm{Atm}^{-/-}$mice. (b) Bands of MDA-protein adducts for $\mathrm{Atm}^{+/+}$and $\mathrm{Atm}^{-/-}$astrocytes. (c) Bands of phosphorylated and total ERK1/2 of $\mathrm{Atm}^{+/+}$and $\mathrm{Atm}^{-/-}$astrocytes are shown. The relative levels of ERK1 and ERK2 phosphorylation were normalized by the levels of total ERK1 and ERK2, respectively, and the average phosphorylation levels are shown in the bars below the Western blotting graphs. *identifies $P<0.05$, average phosphorylation of ERK1/2 in $\mathrm{Atm}^{+/+}$vs that in $\mathrm{Atm}^{-1-}$. (d) Bands of phosphorylated and total ERK1/2 of $\mathrm{Atm}^{-1-}$ astrocytes are shown by Western blotting. The relative levels of ERK1 and ERK2 phosphorylation were normalized by the levels of total ERK1 and ERK2, respectively, and the average phosphorylation levels are shown in the bars below the Western blotting graphs. *identifies $P<0.05$, average phosphorylation of ERK1/2 in $\mathrm{Atm}^{-1-}$ vs that with NAC treatment.

much higher in $\mathrm{Atm}^{-1-}$ astrocytes than in $\mathrm{Atm}^{+/+}$ astrocytes (Figure $3 \mathrm{~b}$ ). Together, these data indicate that ATM deficiency induces oxidative stress responses in astrocytes.
We have shown previously that ER stress is associated with oxidative stress in astrocytes infected by a cytopathic retrovirus-ts $1 .{ }^{22,38}$ Here, we have shown that levels of ER stress markers, GRP78 and cleavage of procaspase-12 were also increased in $\mathrm{Atm}^{-1-}$ astrocytes (Figure 3a).

\section{ATM Deficiency Induces Redox-Sensitive ERK1/2 Phosphorylation in Astrocytes}

Mitogen-activated protein kinase (MAPK) subfamilies include ERK1/2, the c-Jun $\mathrm{NH}_{2}$-terminal kinases (JNK), and the p38 kinases. Here we showed that the levels of phosphorylated ERK1/2 increased in $\mathrm{Atm}^{-1-}$ astrocytes (2.6-fold for ERK1 and 1.7-fold for ERK2 compared with the levels in $\mathrm{Atm}^{+/+}$astrocytes, Figure 3c). JNK and p38, however, were not detectable in these astrocytes. Recently, it has been shown that the MAPK/ERK stress pathway is redox sensitive. ${ }^{39-41}$ To determine whether the increase in ERK1/2 phosphorylation and activation is a consequence of oxidative stress, we treated $\mathrm{Atm}^{-/-}$ astrocytes with antioxidant $\mathrm{N}$-acetylcyste (NAC). The activation of ERK1/2 in these astrocytes was suppressed by treatment with NAC $(38 \%$ reduction for ERK1 and 36\% reduction for ERK2, Figure 3d). This suggests that astrocytes respond to the ATMdeficiency-induced oxidative stress by activating the redox-sensitive ERK1/2 pathways.

\section{ATM Deficiency Increases HSP70 Levels and ERK1/2 Phosphorylation in the Cerebellum}

Humans with A-T show progressive deterioration of cerebellar functions and development of severe ataxia. In $\mathrm{Atm}^{-/-}$mice, increased oxidative stress in the cerebellum has been reported. ${ }^{7}$ In this study, we found that HSP70 levels in cerebella of 2-monthold $\mathrm{Atm}^{-1-}$ mice were much higher than those of their $\mathrm{Atm}^{+/+}$littermates (Figure 4a, $P<0.01$ ). In addition, the levels of phosphorylated ERK1/2 were also higher in the cerebella of $\mathrm{Atm}^{-1-}$ mice than in those of $\mathrm{Atm}^{+/+}$mice (Figure 4b, P<0.01).

\section{Phosphorylated ERKs is Increased in Bergmann Glia in the Atm ${ }^{-1-}$ Cerebellum}

In the cerebellum, Bergmann glia are in direct contact with Purkinje cells. We observed that the levels of phosphorylated ERKs (including ERK1 and ERK2) staining was increased primarily in the Bergmann glia from $\mathrm{Atm}^{-1-}$ cerebella of 2-monthold mice, with degenerating Purkinje neurons nearby (Figure 5b). This was not seen in $\mathrm{Atm}^{+/+}$ cerebella (Figure 5a). These findings suggest that oxidative stress occurs in astrocytes in the cerebella of $\mathrm{Atm}^{-1-}$ mice. The $\mathrm{Atm}^{-1-}$ Purkinje cell degeneration near the stressed astrocytes suggests that 
Purkinje cell degeneration may be the result of the oxidatively stressed $\mathrm{Atm}^{-1-}$ astrocytes. Bergmann glia next to Purkinje cells were labeled by $\mathrm{S} 100$ antibody (Figure 5c) and GFAP staining (Figure 5d), which show identical localization in serial tissue sections.

Signs of oxidative stress were also present in other areas of the brain from 3-month-old $\mathrm{Atm}^{-1-}$ mice, for example the cortex. Phosphorylation and nuclear translocation of ERKs were observed in cortical neuronal cells (Figure 6b), and cortical sections showed signs of astroglios (Figure 7b), possibly due to astrocytic response to neuronal damage.
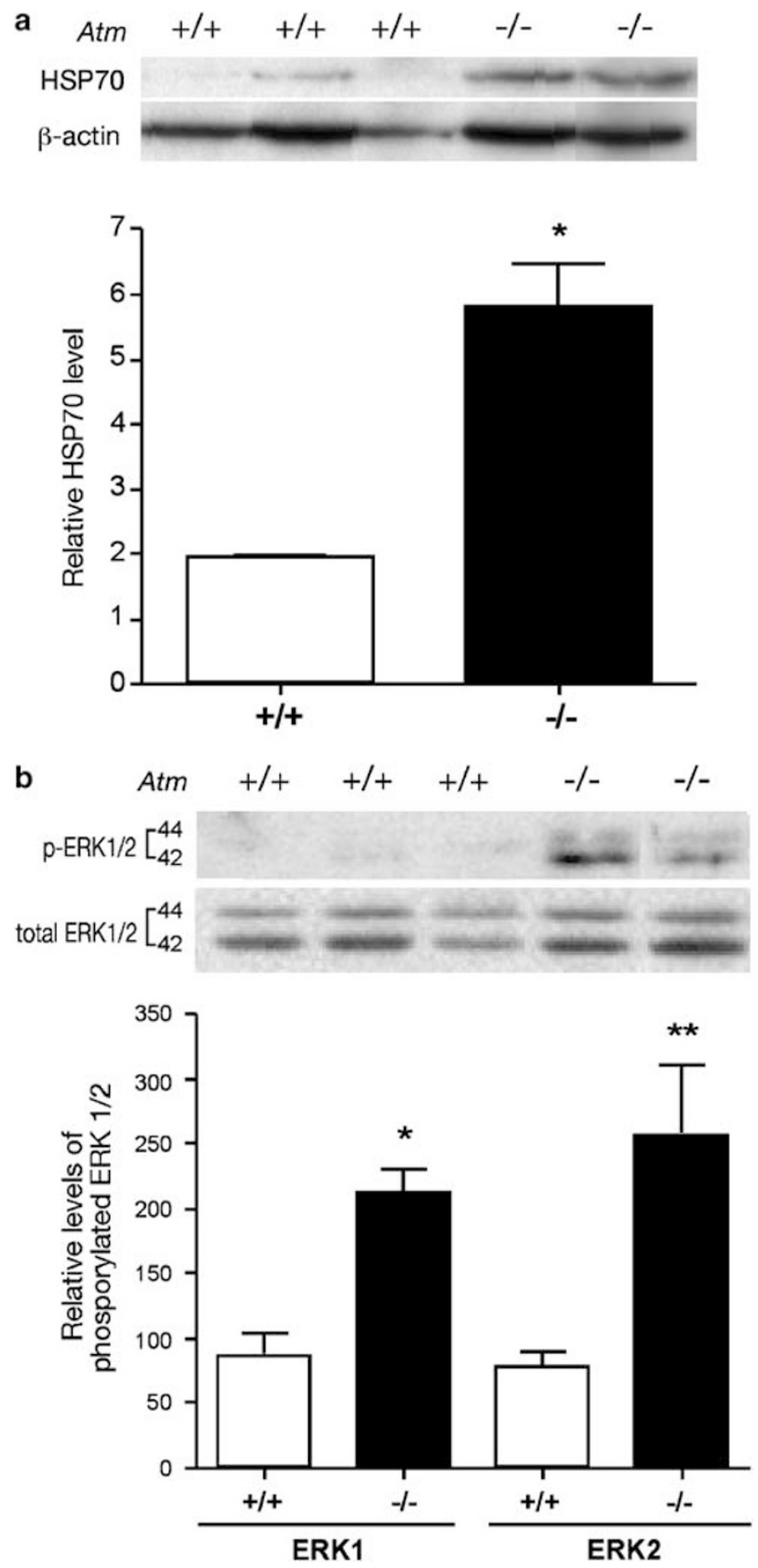

\section{Discussion}

Primary astrocytes isolated from $\mathrm{Atm}^{-1-}$ mice show growth abnormality in culture, as do other primary cells isolated from humans with A-T or from $\mathrm{Atm}^{-/-}$ mice. ${ }^{26,31,32,42-45}$ Our study here also shows spontaneous DNA synthesis in primary $\mathrm{Atm}^{-/-}$astrocytes, which is consistent with our previous findings that DNA synthesis is markedly increased in $\mathrm{Atm}^{-1-}$ mouse thymocytes and in ATM-deficient human lymphocytes, ${ }^{26,32}$ which is associated with increased intracellular levels of $\mathrm{H}_{2} \mathrm{O}_{2}$ (data not shown). $\mathrm{H}_{2} \mathrm{O}_{2}$ induced DNA synthesis also occur in thymocytes from another murine model of genetic cellular antioxidant deficiency. ${ }^{46}$ Spontaneous DNA synthesis and the expression of certain markers of oxidative stress, observed in our thymocyte studies and reported here in $\mathrm{Atm}^{-1-}$ astrocytes, suggest that ATM deficiency may affect cells from multiple anatomical systems, notably the immune and nervous systems, in similar ways.

Although overt signs of degeneration in the $\mathrm{Atm}^{-1-}$ CNS have been a subject of controversy, there is now a growing body of evidence showing oxidative stress in ATM-deficient CNS cells, which disposes the ATMdeficient brain to degeneration. Others have shown that neural precursor cells cultured in lower concentrations of FBS produce higher levels of $\mathrm{ROS},{ }^{47}$ and that at higher concentrations of FBS (which contains antioxidants) ROS production is reduced. ${ }^{46}$ Studies on $\mathrm{Atm}^{-1-}$ thymocytes also show that higher FBS concentrations, ${ }^{46}$ or other antioxidants, such as $\mathrm{NAC}^{26}$ and $\alpha$-lipoic acid (data not shown), suppress spontaneous DNA synthesis by counteracting rising intracellular $\mathrm{H}_{2} \mathrm{O}_{2}$ levels. ${ }^{48}$ Since spontaneous DNA synthesis in $\mathrm{Atm}^{-1-}$ cells can be suppressed by antioxidant treatment, it is likely that this increased spontaneous DNA synthesis is a cellular response to oxidative stress in $\mathrm{Atm}^{-1-}$ astrocytes. However, this increased spontaneous DNA synthesis in $\mathrm{Atm}^{-1-}$ astrocytes does not lead to cell proliferation, as shown by the fact that $\mathrm{Atm}^{-/-}$astrocytes do not show increased numbers of cells in S-phase. Further investigation on this subject is warranted and is currently underway in our laboratory.

Figure 4 HSP70 and phosphorylated ERK1/2 levels are increased in $\mathrm{Atm}^{-1-}$ cerebella. In all 2-month-old mice were used for cerebella dissection and protein extraction before thymic lymphoma developed. Three $\mathrm{Atm}^{+/+}$mice and two $\mathrm{Atm}^{-1-}$ mice were used in this experiment. The bands in the blots were scanned for density analysis. The graphs represent two separate experiments. (a) Bands of HSP70 of the cerebella. The related levels of HSP70 were normalized by $\beta$-actin and the average levels of HSP 70 were shown in the bars below the Western blotting graphs. *identifies $P<0.01$, average HSP70 level in $\mathrm{Atm}^{-1-} v s$ that in $\mathrm{Atm}^{+/+}$. (b) The bands of phosphorylated ERK1/2 and total ERK1/2 in the cerebella. The relative levels of phosphorylated ERK1 and ERK2 were normalized by the total levels of ERK1 and ERK2, respectively, and the average phosphorylation levels are shown in the bars below the Western blotting graphs. *identifies $P<0.05$, average phosphorylation of ERK1 in $\mathrm{Atm}^{-1-}$ vs that in Atm $^{+/+},{ }^{* *}$ identifies $P<0.05$ for ERK2. 


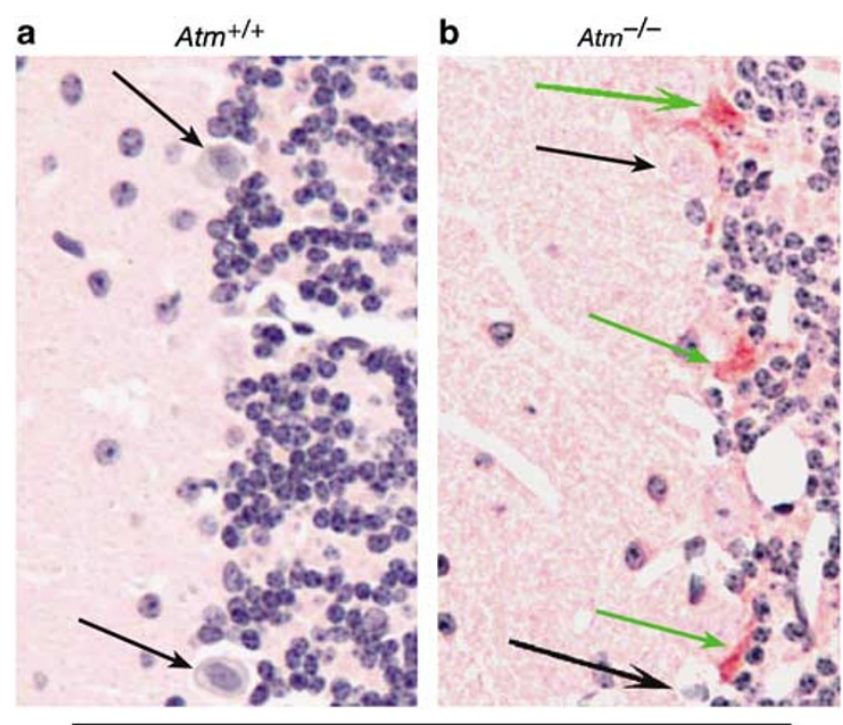

P-ERK

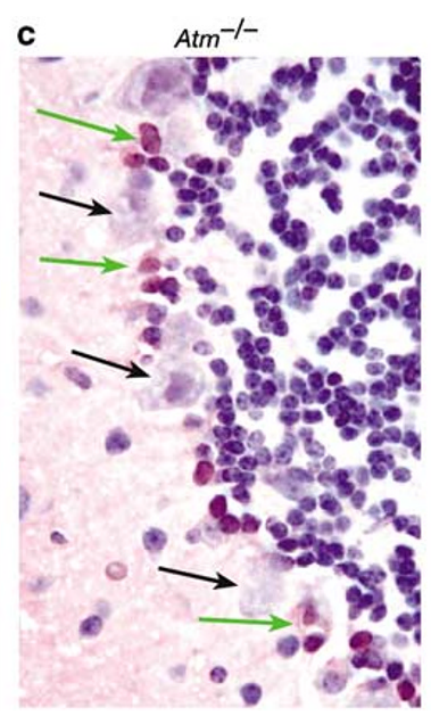

$\mathrm{S} 100$

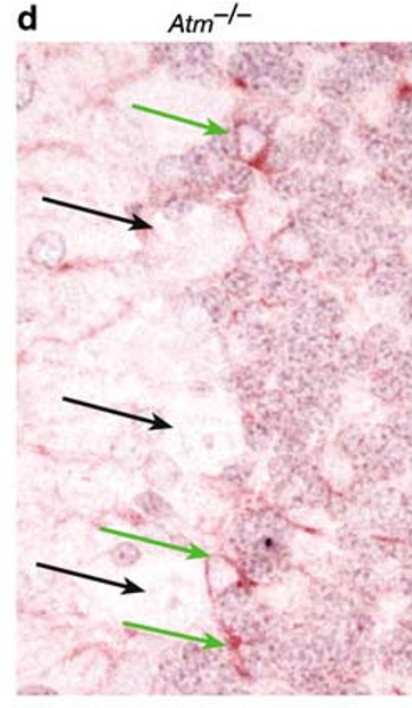

GFAP

Figure 5 Expression of phosphorylated ERKs in the cerebella of 2-month-old $\mathrm{Atm}^{+/+}$and $\mathrm{Atm}^{-/-}$mice. (a) No phosphorylated ERKs staining was detected in the cerebellum of an $\mathrm{Atm}^{+/+}$mouse. Black arrows denote normal Purkinje cells. (b) Phosphorylated ERKs staining (red) was found primarily in Bergmann glia in the cerebellum of an $\mathrm{Atm}^{-1-}$ mouse. Green arrows identify Bergmann glia with positive staining for phosphorylated ERKs, and the black arrows identify degenerated Purkinje cells. (c) S100 staining in Bergmann glia in the cerebellum of the serial section of the same $\mathrm{Atm}^{-1-}$ mouse. Green arrows identify Bergmann glia with positive staining for S100. (d) GFAP staining in Bergmann glia in the cerebellum of the serial section of the same $\mathrm{Atm}^{-/-}$mouse. Green arrows identify Bergmann glia with positive staining for GFAP. Block arrows in (c) and (d) identify degenerated Purkinje cells. The similar staining differences were seen from the serial sections of the cerebella of three $\mathrm{Atm}^{-1-}$ and three $\mathrm{Atm}^{+/+}$mice. Magnification $\times 200$.


Figure 6 Expression of phosphorylated ERKs in the cerebral cortex of one 3-month-old $\mathrm{Atm}^{+/+}$and $\mathrm{Atm}^{-/-}$mice. Notice the absence of labeled cells on (a) $\mathrm{Atm}^{+1+}$ compared with (b) $\mathrm{Atm}^{-1-}$ that shows neuronal nuclear labeling. Magnification $\times 200$.

We found that many markers of oxidative stress, including MDA-protein adducts, MnSOD, $\mathrm{Cu} / \mathrm{ZnSOD}$, and HSP70, were upregulated in these primary $\mathrm{Atm}^{-1-}$ astrocytes, ER stress markers (GRP78 and caspase 12 cleavage) were also upregulated, and the redox-sensitive ERK1/2 was highly activated. Since the activation of ERK1/2 and spontaneous DNA synthesis were suppressed by NAC and high concentrations $(40 \%)$ of FBS, we suggest that ATM functions to maintain redox and energy homeostasis in cultured astrocytes. In the absence of ATM, the oxidatively stressed astrocytes respond by activating their oxidative stress responses, including the heat shock response, the redox-sensitive signaling pathway (ERK1/2), and the ER stress response. As we also observed similar HSP70 and ERK1/2 responses in the cerebella of $\mathrm{Atm}^{-/-}$mice, we conclude that what we observed in astrocytes in vitro also occurs in vivo.

It is not clear how oxidative stress is induced in ATM deficiency. Some studies have shown that ATM deficiency causes depletion of antioxidants, 

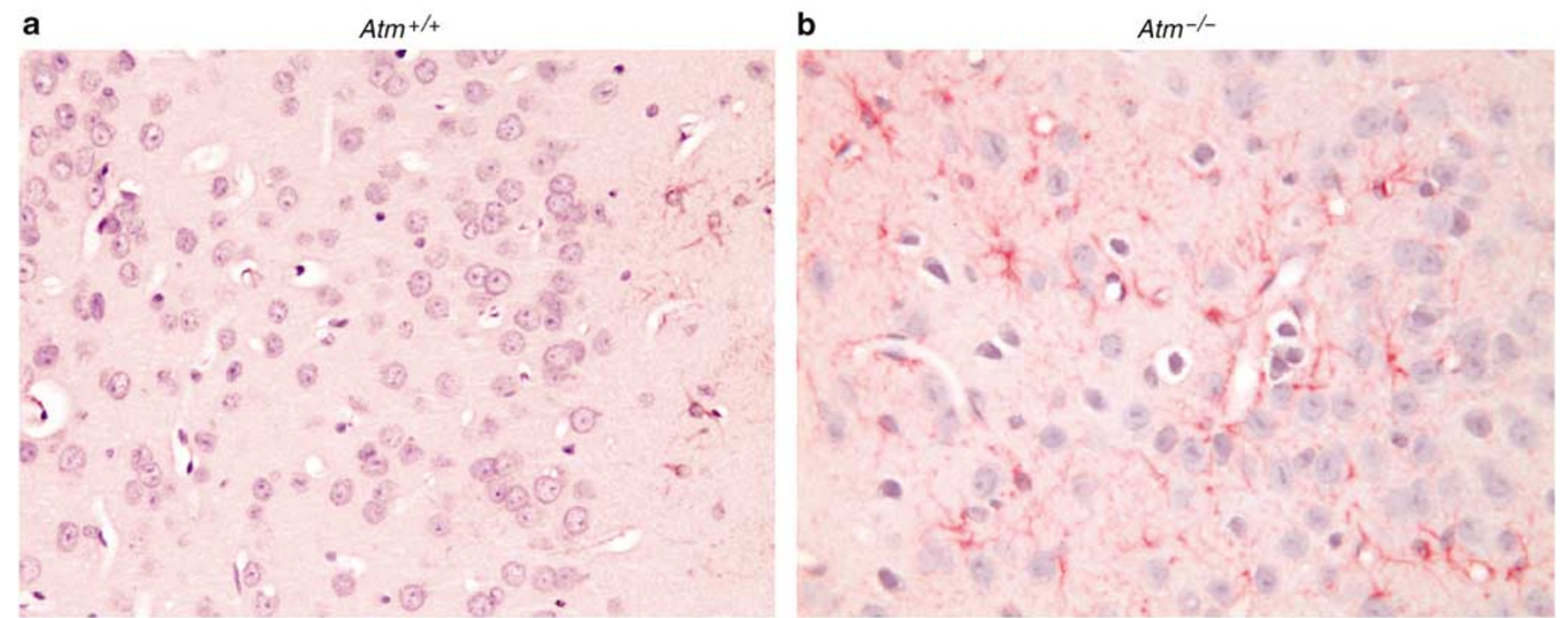

Figure 7 Expression of GFAP labeled astrocytes in the cerebral cortex of one 3-month-old Atm $^{+/+}$and Atm $^{-/-}$mice. Notice that in the (a) $\mathrm{Atm}^{+/+}$there are only few labeled astrocytes in the external molecular layer compared with (b) Atm ${ }^{-/-}$where there is an abundant of labeled activated astrocytes. Magnification $\times 200$.

including glutathione, ${ }^{49}$ peroxiredoxin $\mathrm{I}^{40}$ and $\mathrm{NAD}^{+} / \mathrm{NADH}^{2,50}$ Antioxidant deficiency renders cells more vulnerable to oxidative stress, leading to DNA oxidative damage. ${ }^{50}$ Treatment with antioxidants has been shown to reduce oxidative stress and DNA damage in ATM deficiency, ${ }^{50}$ to protect Purkinje cells from oxidative stress-induced degeneration, ${ }^{9}$ and to ameliorate neurobehavioral defects in $\mathrm{Atm}^{-1-}$ mice. ${ }^{8}$

We show here for the first time that ER stress signals are also elevated in $\mathrm{Atm}^{-1-}$ astrocytes. The ER is a membranous network that requires a proper oxidative environment for folding newly synthesized proteins, and ER stress can be caused by conditions that perturb the oxidative environment and protein folding process as a result of energy deprivation and redox imbalance. ${ }^{51-53}$ Thus it is likely that the ER stress response seen in $\mathrm{Atm}^{-1-}$ astrocytes is a result of cellular redox imbalance.

Another novel finding from this study is the involvement of the MAPK/ERK signaling pathway in $\mathrm{Atm}^{-1-}$ astrocytes. MAPK signaling pathways have been shown to play important roles in apoptosis, oncogenic transformation, and inflammatory or oxidative stress responses in various cell types. ${ }^{54}$ Among the three major mammalian MAPK subfamilies, the ERK signaling pathway is most sensitive to oxidative stress. Activation of ERK by growth factors or oxidants can promote either cell proliferation ${ }^{55-57}$ or cell death, especially in neuronal cells. ${ }^{58-61}$ These effects can be blocked by antioxidants. ${ }^{62}$ Recent work by others also shows marked ERK1/2 activation in oxidatively stressed neurons. ${ }^{63}$

In our study, ERK phosphorylation/activation was found in primary cultured $\mathrm{Atm}^{-/-}$astrocytes, and this activation was markedly suppressed by treatment with antioxidant NAC. Therefore, we con- cluded that the ERK activation was probably due to the oxidative stress caused by ATM deficiency. We also noted increased ERK phosphorylation of Bergmann glia near degenerating Purkinje neurons in $\mathrm{Atm}^{-1-}$ mice. Bergmann glia are important in the development and support of Purkinje neurons. Thus, it is likely that oxidative stress, as indicated by ERK activation in $\mathrm{Atm}^{-/-}$astrocytes, may lead to astrocyte dysfunction, partially accounting for the degeneration of nearby neurons in the cerebellum.

Interestingly, we also found increased ERK phosphorylation and nuclear translocation in cortical neurons, dopaminergic neurons, and neurons in other brain regions including the olfactory bulb (data not shown) from older $\mathrm{Atm}^{-/-}$mice, which indicate wide distribution of neuronal oxidative stress in the CNS in $\mathrm{Atm}^{-1-}$ mice. Although neurons are generally believed to be in the quiescent state in vivo, a recent study shows that $\mathrm{Atm}^{-1-}$ neurons undergo cell cycle re-entry, which could eventually lead to neuronal death. ${ }^{13}$ This and other signs of 'predegenerative' CNS lesions, including age-dependent neuronal cell loss and gliosis and dystrophic Purkinje cells, indicate that Purkinje cells and other neurons are damaged both in A-T humans and in ATM-deficient mice. Parallels between the pathologies of thymocytes from A-T humans and ATM-deficient mice have also been reported in our previous studies. It appears that in different cell types (thymocytes, astrocytes and neurons), ATM deficiency may initiate a common cellular mechanism to activate DNA synthesis and oxidative stress, leading to cell deficiency in proliferation, development, differentiation and maintaining normal physiologic functions. Whether this is the case in $\mathrm{Atm}^{-/-}$neurons is now under investigation in our laboratory.

In summary, our previous data on primary thymocytes and present data on primary astrocytes 
have shown that the absence of ATM leads to intrinsic defects in these cells, which is manifested as unchecked DNA synthesis, failure of normal growth, differentiation and development, and abnormal levels of cell death, without ionizing radiation or other DNA damage agents. In $\mathrm{Atm}^{-1-}$ thymocytes, lack of ATM leads to early thymic atrophy and later to thymic lymphomas in the $\mathrm{Atm}^{-/-}$mice, due to the abnormal development and neoplastic transformation in the few thymocytes that survive these defects in the $\mathrm{Atm}^{-/-}$thymus. ${ }^{25,26}$ As reported here, the abnormalities in primary $\mathrm{Atm}^{-1-}$ astrocytes, including (a) inability to grow and develop normally, (b) increased spontaneous DNA synthesis, and (c) oxidative stress, ER stress and redox-sensitive ERK1/2 response, possibly contribute to the inability to provide normal support (in particular redox support) to nearby neurons in vivo. Use of antioxidants to reduce oxidative stress, especially in oxidation-hypersensitive organs such as the brain, may be therapeutically effective in ATM deficiency.

\section{Acknowledgements}

We thank Vanessa Edwards, Shawna Johnson, David Galloway, and Cynthia Kim for their assistance in preparing the manuscript and Lifang Zhang, Shoufeng Wang, and Amy Reid for their technical support. We thank Dr Jiangjun Shen of the Molecular Biology Core and Kent Claypool of the Cell and Tissue Analysis Facility Core at Science Park. We are also most grateful to the support of Robert Howard and the Longevity Foundation (formerly A-T Project, Austin, TX). This work was also supported by Center Grant ES07784 and Core Grant CA 16672, both from the National Institutes of Health.

\section{References}

1 Abraham RT. Cell cycle checkpoint signaling through the ATM and ATR kinases. Genes Dev 2001;15:2177-2196.

2 Barzilai A, Rotman G, Shiloh Y. ATM deficiency and oxidative stress: a new dimension of defective response to DNA damage. DNA Repair (Amsterdam) 2002;1:3-25.

3 McKinnon PJ. ATM and ataxia telangiectasia. EMBO Rep 2004;5:772-776.

4 Shiloh Y. ATM and ATR: networking cellular responses to DNA damage. Curr Opin Genet Dev 2001; 11:71-77.

5 Ito K, Hirao A, Arai F, et al. Regulation of oxidative stress by ATM is required for self-renewal of haematopoietic stem cells. Nature 2004;431:997-1002.

6 Barlow C, Dennery PA, Shigenaga MK, et al. Loss of the ataxia-telangiectasia gene product causes oxidative damage in target organs. Proc Natl Acad Sci USA 1999; 96:9915-9919.

7 Kamsler A, Daily D, Hochman A, et al. Increased oxidative stress in ataxia telangiectasia evidenced by alterations in redox state of brains from Atm-deficient mice. Cancer Res 2001;61:1849-1854.

8 Browne SE, Roberts II LJ, Dennery PA, et al. Treatment with a catalytic antioxidant corrects the neurobehavioral defect in ataxia-telangiectasia mice. Free Radic Biol Med 2004;36:938-942.

9 Chen P, Peng C, Luff J, et al. Oxidative stress is responsible for deficient survival and dendritogenesis in Purkinje neurons from ataxia-telangiectasia mutated mutant mice. J Neurosci 2003;23:11453-11460.

10 Quick KL, Dugan LL. Superoxide stress identifies neurons at risk in a model of ataxia-telangiectasia. Ann Neurol 2001;49:627-635.

11 Eilam R, Peter Y, Groner Y, et al. Late degeneration of nigro-striatal neurons in ATM-/- mice. Neuroscience 2003;121:83-98.

12 Kuljis RO, $\mathrm{Xu} \mathrm{Y,} \mathrm{Aguila} \mathrm{MC,} \mathrm{et} \mathrm{al.} \mathrm{Degeneration} \mathrm{of}$ neurons, synapses, and neuropil and glial activation in a murine Atm knockout model of ataxia-telangiectasia. Proc Natl Acad Sci 1997;94:12688-12693.

13 Yang Y, Herrup K. Loss of neuronal cell cycle control in ataxia-telangiectasia: a unified disesase mechanism. J Neurosci 2005;15:2522-2529.

14 Chen Y, Vartiainen NE, Ying W, et al. Astrocytes protect neurons from nitric oxide toxicity by a glutathione-dependent mechanism. J Neurochem 2001;77:1601-1610.

15 Desagher S, Glowinski J, Premont J. Astrocytes protect neurons from hydrogen peroxide toxicity. J Neurosci 1996;16:2553-2562.

16 Mena MA, Casarejos MJ, Carazo A, et al. Glia conditioned medium protects fetal rat midbrain neurons in culture from L-DOPA toxicity. Neuroreport 1996;7:441-445.

17 Shih AY, Johnson DA, Wong G, et al. Coordinate regulation of glutathione biosynthesis and release by Nrf2-expressing glia potently protects neurons from oxidative stress. J Neurosci 2003;23:3394-3406.

18 Atwood WJ, Berger JR, Kaderman R, et al. Human immunodeficiency virus type 1 infection of the brain. Clin Microbiol Rev 1993;6:339-366.

19 Brack-Werner R. Astrocytes: HIV cellular reservoirs and important participants in neuropathogenesis. Aids 1999;13:1-22.

20 Friedlander RM. Apoptosis and caspases in neurodegenerative diseases. N Engl J Med 2003;348:1365-1375.

21 Galey D, Becker K, Haughey N, et al. Differential transcriptional regulation by human immunodeficiency virus type 1 and gp120 in human astrocytes. J Neurovirol 2003;9:358-371.

22 Liu N, Kuang X, Kim HT, et al. Possible involvement of both endoplasmic reticulum- and mitochondria-dependent pathways in MoMuLV-ts1-induced apoptosis in astrocytes. J Neurovirol 2004;10:189-198.

23 Wong PKY, Lynn WS. Neuroimmunodegeneration. EOS J Immunol Immunopharmacol 1997;17:30-35.

24 Kuang X, Yan M, Liu N, et al. Control of Atm-/thymic lymphoma cell proliferation in vitro and in vivo by dexamethasone. Cancer Chemother Pharmacol 2005; 55:203-212.

25 Yan M, Kuang X, Qiang W, et al. Prevention of thymic lymphoma development in Atm-/- mice by dexamethasone. Cancer Res 2002;62:5153-5157.

26 Yan M, Qiang W, Liu N, et al. The ataxia-telangiectasia gene product may modulate DNA turnover and control cell fate by regulating cellular redox in lymphocytes. FASEB J 2001;15:1132-1138. 
27 Lynn WS, Wong PKY. Neuroimmunodegeneration: do neurons and $\mathrm{T}$ cells use common pathways for cell death? FASEB J 1995;9:1147-1156.

28 Shikova E, Lin YC, Saha K, et al. Correlation of specific virus-astrocyte interactions and cytopathic effects induced by $t s 1$, a neurovirulent mutant of Moloney murine leukemia virus. J Virol 1993;67:1137-1147.

29 Liu N, Qiang W, Kuang X, et al. The peroxisome proliferator phenylbutyric acid (PBA) protects astrocytes from ts1 MoMuLV-induced oxidative cell death. J Neurovirol 2002;8:318-325.

30 Wang ZY, Stoltenberg M, Huang L, et al. Abundant expression of zinc transporters in Bergman glia of mouse cerebellum. Brain Res Bull 2005;64:441-448.

31 Gosink EC, Chong MJ, McKinnon PJ. Ataxia telangiectasia mutated deficiency affects astrocyte growth but not radiosensitivity. Cancer Res 1999;59:5294-5298.

32 Lynn WS, Wong PKY. Possible control of cell death pathways in ataxia telangiectasia. A case report. Neuroimmunomodulation 1997;4:277-284.

33 Furuya S, Tabata T, Mitoma J, et al. L-serine and glycine serve as major astroglia-derived trophic factors for cerebellar Purkinje neurons. Proc Natl Acad Sci USA 2000;97:11528-11533.

34 Dringen R, Hirrlinger J. Glutathione pathways in the brain. Biol Chem 2003;384:505-516.

35 Wang XF, Cynader MS. Astrocytes provide cysteine to neurons by releasing glutathione. J Neurochem 2000; 74:1434-1442.

36 Wang XF, Cynader MS. Pyruvate released by astrocytes protects neurons from copper-catalyzed cysteine neurotoxicity. J Neurosci 2001;21:3322-3331.

37 Mazur-Kolecka B, Kowal D, Sukontasup T, et al. The effect of oxidative stress on accumulation of apolipoprotein E3 and E4 in a cell culture model of betaamyloid angiopathy (CAA). Brain Res 2003;983:48-57.

38 Qiang W, Cahill JM, Liu J, et al. Activation of transcription factor Nrf-2 and its downstream targets in response to moloney murine leukemia virus ts1induced thiol depletion and oxidative stress in astrocytes. J Virol 2004;78:11926-11938.

39 El Jamali A, Freund C, Rechner C, et al. Reoxygenation after severe hypoxia induces cardiomyocyte hypertrophy in vitro: activation of CREB downstream of GSK3beta. FASEB J 2004;18:1096-1098.

$40 \mathrm{Li} \mathrm{B}$, Wang X, Rasheed N, et al. Distinct roles of c-Abl and Atm in oxidative stress response are mediated by protein kinase C delta. Genes Dev 2004;18:1824-1837.

41 Mazak I, Fiebeler A, Muller DN, et al. Aldosterone potentiates angiotensin II-induced signaling in vascular smooth muscle cells. Circulation 2004;109: 2792-2800.

42 Barlow C, Hirotsune S, Paylor R, et al. Atm-deficient mice: a paradigm of ataxia telangiectasia. Cell 1996;86: 159-171.

43 Elson A, Wang Y, Daugherty CJ, et al. Pleiotropic defects in ataxia-telangiectasia protein-deficient mice. Proc Natl Acad Sci USA 1996;93:13084-13089.

44 Lavin MF, Shiloh Y. The genetic defect in ataxiatelangiectasia. Annu Rev Immunol 1997;15:177-202.

$45 \mathrm{Xu} \mathrm{Y,} \mathrm{Baltimore} \mathrm{D.} \mathrm{Dual} \mathrm{roles} \mathrm{of} \mathrm{ATM} \mathrm{in} \mathrm{the} \mathrm{cellular}$ response to radiation and in cell growth control. Genes Dev 1996;10:2401-2410.

46 Moon EY, Han YH, Lee DS, et al. Reactive oxygen species induced by the deletion of peroxiredoxin II(PrxII) increases the number of thymocytes resulting in the enlargement of PrxII-null thymus. Eur J Immunol 2004;34:2119-2128.

47 Limoli CL, Rola R, Giedzinski E, et al. Cell-densitydependent regulation of neural precursor cell function. Proc Natl Acad Sci USA 2004;101:16052-16057.

48 Sauer H, Wartenberg M, Hescheler J. Reactive oxygen species as intracellular messengers during cell growth and differentiation. Cell Physiol Biochem 2001;11: 173-186.

49 Meredith MJ, Dodson ML. Impaired glutathione biosynthesis in cultured human ataxia-telangiectasia cells. Cancer Res 1987;47:4576-4581.

50 Reliene R, Fischer E, Schiestl RH. Effect of $N$-acetyl cysteine on oxidative DNA damage and the frequency of DNA deletions in atm-deficient mice. Cancer Res 2004;64:5148-5153.

51 Sitia R, Molteni SN. Stress, protein (mis)folding, and signaling: the redox connection. Sci STKE 2004;239: pe27.

52 Kaufman RJ. Stress signaling from the lumen of the endoplasmic reticulum: coordination of gene transcriptional and translational controls. Genes Dev 1999; 13:1211-1233.

$53 \mathrm{Ma}$ Y, Hendershot LM. The unfolding tale of the unfolded protein response. Cell 2001;107:827-830.

54 Hagemann C, Blank JL. The ups and downs of MEK kinase interactions. Cell Signal 2001;13:863-875.

55 Guyton KZ, Liu Y, Gorospe M, et al. Activation of mitogen-activated protein kinase by $\mathrm{H}_{2} \mathrm{O}_{2}$. Role in cell survival following oxidant injury. J Biol Chem 1996; 271:4138-4142.

56 Wong PKY, Lynn WS, Lin YC, et al. ts1 MoMuLV: a murine model of neuroimmunodegeneration. In: Wong PKY, Lynn WS (eds). Neuroimmunodegeneration. R.G. Landes: Heidelberg, 1998, pp 75-93.

57 Xia Z, Dickens M, Raingeaud J, et al. Opposing effects of ERK and JNK-p38 MAP kinases on apoptosis. Science 1995;270:1326-1331.

58 Bhat NR, Zhang P. Hydrogen peroxide activation of multiple mitogen-activated protein kinases in an oligodendrocyte cell line: role of extracellular signalregulated kinase in hydrogen peroxide-induced cell death. J Neurochem 1999;72:112-119.

59 Lesuisse C, Martin LJ. Immature and mature cortical neurons engage different apoptotic mechanisms involving caspase-3 and the mitogen-activated protein kinase pathway. J Cereb Blood Flow Metab 2002;22: 935-950.

60 Noshita N, Sugawara T, Hayashi T, et al. Copper/zinc superoxide dismutase attenuates neuronal cell death by preventing extracellular signal-regulated kinase activation after transient focal cerebral ischemia in mice. J Neurosci 2002;22:7923-7930.

61 Stanciu M, Wang Y, Kentor R, et al. Persistent activation of ERK contributes to glutamate-induced oxidative toxicity in a neuronal cell line and primary cortical neuron cultures. J Biol Chem 2000; 275:12200-12206.

62 Subramaniam S, Zirrgiebel U, von Bohlen Und Halbach $\mathrm{O}$, et al. ERK activation promotes neuronal degeneration predominantly through plasma membrane damage and independently of caspase-3. J Cell Biol 2004;165:357-369.

63 Chu CT, Levinthal DJ, Kulich SM, et al. Oxidative neuronal injury: the dark side of ERK1/2. Eur J Biochem 2004;271:2060-2066. 